\title{
Intramolecular excimer and exciplex emission of 1,4-dipyrenyl substituted cyclohexasilane
}

\author{
Cornelis A. van Walree ${ }^{a, *}$, Veronica E.M. Kaats-Richters ${ }^{a}$, \\ Leonardus W. Jenneskens ${ }^{a}$, René M. Williams ${ }^{b}$, Ivo H.M. van Stokkum ${ }^{c}$ \\ ${ }^{a}$ Debye Institute, Department of Physical Organic Chemistry, Utrecht University, Padualaan 8, 3584 CH Utrecht, The Netherlands \\ b Molecular Photonic Materials, Institute of Molecular Chemistry, Faculty of Science, University of Amsterdam, \\ Nieuwe Achtergracht 166, 1018 WV Amsterdam, The Netherlands \\ ${ }^{\mathrm{c}}$ Faculty of Sciences, Vrije Universiteit Amsterdam, De Boelelaan 1081, 1081 HV Amsterdam, The Netherlands
}

Received 6 December 2001

\begin{abstract}
Intramolecular excimer emission is observed for cis-1,4-di(1-pyrenyl)decamethylcyclohexasilane in nonpolar solvents. Time-resolved fluorescence spectroscopy and kinetic modelling indicate that the driving force of excimer formation is very small, and that the process is governed by the flexibility of the silicon ring. In the polar solvent acetonitrile, photoinduced electron transfer occurs, with the cyclohexasilane ring acting as electron donor and the pyrenyl group as electron acceptor. (c) 2002 Elsevier Science B.V. All rights reserved.
\end{abstract}

\section{Introduction}

The study of interactions between photoactive units can yield valuable information on the medium between these units. Hence, attachment of the textbook chromophore pyrene to oligosilanes gives insight into the intriguing electronic and conformational properties of these catenates of silicon atoms. These oligosilanes are said to be $\sigma$-conjugated as their electronic transitions occur in the near UV spectral region, they interact

\footnotetext{
${ }^{*}$ Corresponding author. Fax: +31-302534533.

E-mail address: c.a.vanwalree@chem.uu.nl (C.A. van Walree).
}

with aromatic moieties via $\sigma-\pi$ mixing and have relatively low ionization potentials [1,2]. Aryl substituted linear oligosilanes have been found to exhibit interesting properties such as TICT-like dual fluorescence [3] and intramolecular excimer emission [4,5]. In contrast, the electronic and photophysical behaviour of derivatives of cyclic oligosilanes is still undisclosed. Here we report on the surprising photophysical properties of the cyclohexasilane derivatives 1-pyrenylundecamethylcyclohexasilane (1) and cis-1,4-di(1-pyrenyl)decamethylcyclohexasilane (2, Scheme 1). X-ray diffraction has shown that in the crystalline state the cyclohexasilane ring of 2 possesses a boat-like structure with the pyrenyl substituents adopting an equatorial or bisectional position [6]. 

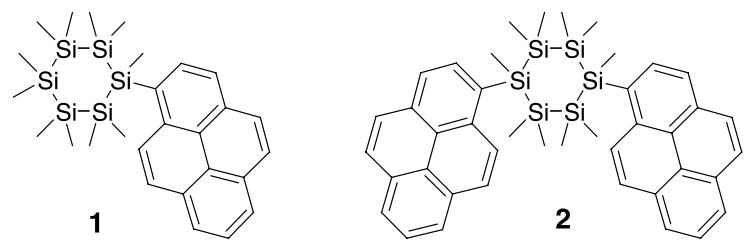

Scheme 1.

\section{Experimental}

Details on the synthesis of $\mathbf{1}$ and $\mathbf{2}$ will be published separately [6]. Steady-state UV and fluorescence spectra were recorded on Cary 1 and Spex Fluorolog instruments, respectively [7]. Time-resolved fluorescence spectra were collected on streak camera [8] or optical multichannel analyzer [9] systems described elsewhere. For all fluorescence measurements samples were degassed by purging with argon for $15 \mathrm{~min}$. Cyclic voltammetry was conducted with an EG\&GPAR model 63A potentiostat/galvanostat in acetonitrile following a procedure described previously [7].

\section{Results and discussion}

UV spectra of $\mathbf{1}$ and $\mathbf{2}$ in cyclohexane are shown in Fig. 1. Apart from intensity differences reflecting the number of pyrenyl chromophores, the spectra of $\mathbf{1}$ and $\mathbf{2}$ are essentially identical. They are strongly related to the spectrum of pyrene, al-

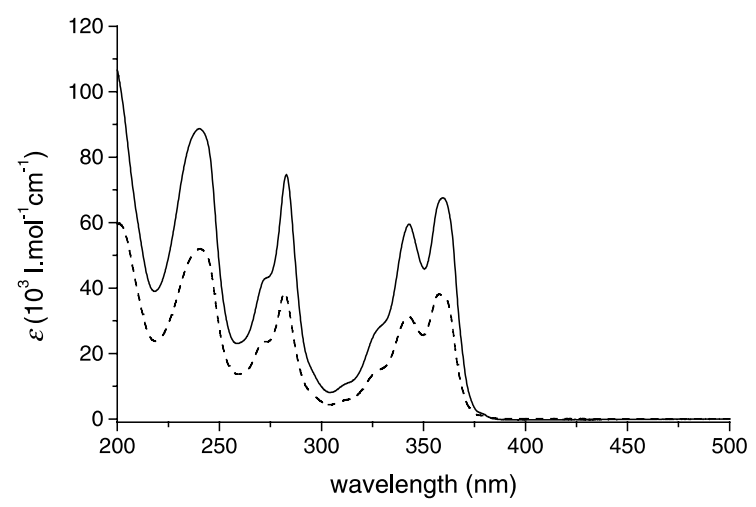

Fig. 1. UV-spectra of di- and monopyrenyl substituted cyclohexasilanes $\mathbf{1}$ (dashed) and $\mathbf{2}$ (solid) in cyclohexane. Maxima of both spectra are essentially identical and are situated at 359, 343,282 and $240-241 \mathrm{~nm}$. though peaks are broadened and the ${ }^{1} \mathrm{~L}_{\mathrm{a}}$ and $\mathrm{B}_{\mathrm{b}}$ transitions have shifted from 335 and $273 \mathrm{~nm}$ in pyrene to 359 and $282 \mathrm{~nm}$, respectively. These shifts are indicative of substantial $\sigma-\pi$ mixing between the silicon ring and the pyrenyl moieties. Interestingly, as inferred from the ${ }^{1} \mathrm{~L}_{\mathrm{a}}$ band position the $\sigma-\pi$ interaction is stronger than in 1-(1-pyrenyl)tridecamethylhexasilane $\left({ }^{1} \mathrm{~L}_{\mathrm{a}}\right.$ maximum $351 \mathrm{~nm}$ ) and related mono- or dipyrenyl substituted linear oligosilanes [4,5]. The strong similarity of the UV spectra of $\mathbf{1}$ and $\mathbf{2}$ shows that in 2 no ground state interaction between the two pyrenyl substituents is operative. A significant solvent effect on the spectra was not detected.

In cyclohexane, monosubstituted 1 exhibits common pyrene-like fluorescence emission with a maximum situated at $381 \mathrm{~nm}$ (Fig. 2). For the dipyrenyl compound an additional broad and weak band in the wavelength range $420-550 \mathrm{~nm}$ is observed. Subtraction of the (normalized) spectrum of $\mathbf{1}$ from that of $\mathbf{2}$ gives a maximum of 458 $\mathrm{nm}$ for this band. No concentration effect on the position and shape of the fluorescence spectrum of 2 (as well as of 1) was noticed in the range $10^{-7}-10^{-4} \mathrm{~mol}^{-1}$. Changing from cyclohexane to medium polarity solvents such as diethyl ether neither did have an effect on the fluorescence

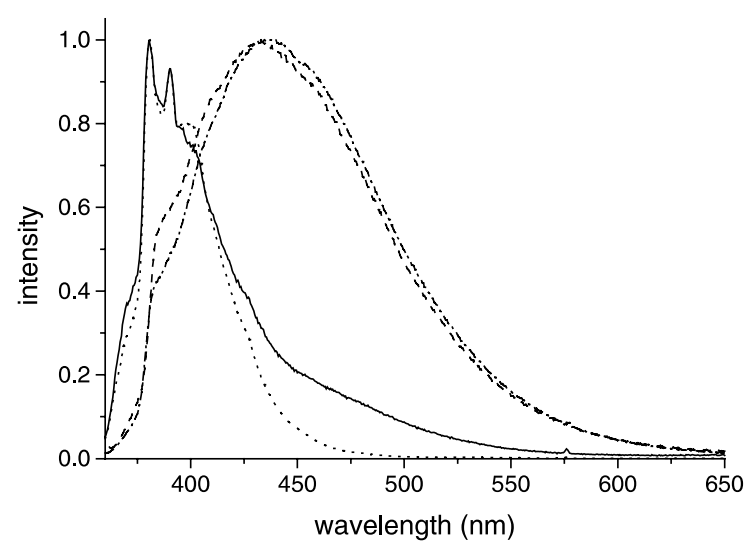

Fig. 2. Fluorescence spectra of $\mathbf{1}$ and 2: (dotted line) $\mathbf{1}$ in cyclohexane; (dash-dotted line) $\mathbf{1}$ in acetonitrile; (solid line) $\mathbf{2}$ in cyclohexane; (dashed line) $\mathbf{2}$ in acetonitrile. Note that the spectra were normalized at their maximum and that the fluorescence in acetonitrile is actually much weaker than in cyclohexane. Concentrations were in the order of $10^{-6} \mathrm{~mol} \mathrm{l}^{-1}$. Excitation wavelength $355 \mathrm{~nm}$. 
spectra. However, as outlined below, in the polar solvent acetonitrile the fluorescence behaviour is different.

Global analysis of time-resolved fluorescence data in cyclohexane, as recorded with a streak camera system, revealed that the fluorescence of 1 decays with a single time constant of $35 \mathrm{~ns}$ over the entire emission band. Fluorescence decays of $\mathbf{2}$ at two wavelengths are shown in Fig. 3, while the Decay Associated Spectra (DAS) are depicted in Fig. 4a. According to the global analysis the decay near $400 \mathrm{~nm}$ is biexponential with lifetimes of 14 and $50 \mathrm{~ns}$. At $460-540 \mathrm{~nm}$, the fluorescence still decays with a $50 \mathrm{~ns}$ time constant, while the $14 \mathrm{~ns}$ component has a negative amplitude and describes the ingrowth of the fluorescence. Hence, the species emitting at long wavelengths is formed out of the species emitting at short wavelengths with this apparent time constant. The observation that the $50 \mathrm{~ns}$ component describes the fluorescence decay at both 402 and $465 \mathrm{~nm}$ suggests that an equilibrium is present between the species emitting at these wavelengths.
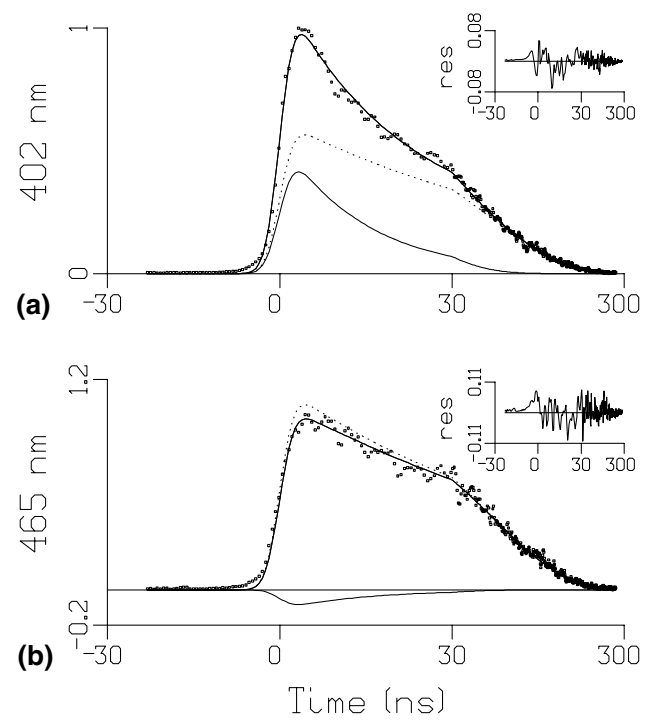

Fig. 3. Fluorescence traces and global fits of 2 in cyclohexane at 402 (a) and 465 (b) $\mathrm{nm}$. The solid and dotted lines represent the 14 and $50 \mathrm{~ns}$ components, respectively. The sum of the contributions is given by the solid line through the measured points. The insets show the residuals of the fits. Note that the abscissa is linear up to $30 \mathrm{~ns}$, and logarithmic above $30 \mathrm{~ns}$.
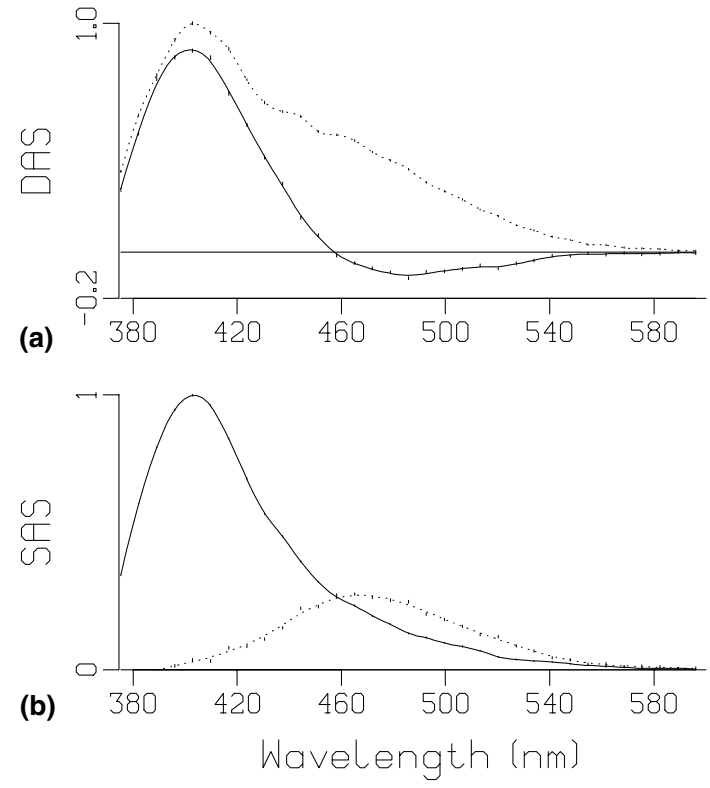

Fig. 4. DAS (a) and Species Associated Spectra (SAS, b) of 2 in cyclohexane obtained from a two-state model (Fig. 5) with the rates $k_{12}$ and $k_{21}$ set equal $\left(\Delta G_{\mathrm{EF}}=0\right)$. Vertical bars indicate estimated standard errors.

All fluorescence data indicate that the broad fluorescence band of $\mathbf{2}$ can be attributed to intramolecular excimer emission, i.e. the two pyrenyl substituents form an excited state complex [10,11]. The formation of the excimer must involve a conformational change of the silicon ring putting both pyrenyl groups into an axial position (folding), which allows them to form the typical faceto-face complex. It is noteworthy that such a folding process can only be imagined to occur for a cis-disubstituted cyclic compound; in a trans-isomer formation of a face-to-face complex is not possible. To the best of our knowledge intramolecular excimer formation has only been reported for linear chain bichromophoric compounds. The dipyrenyl substituted cyclohexasilane 2 thus represents the first intramolecular excimer containing a cyclic spacer ${ }^{1}$.

In comparison to most pyrene-based excimers $[4,5,11,13]$, the energy difference between the

\footnotetext{
${ }^{1}$ Intermolecular excimer emission has been reported for pyrenyl-functionalized trans-1,2-substituted cyclohexanes [12].
} 
monomer and excimer fluorescence for $\mathbf{2}$ is relatively small $\left(4400 \mathrm{~cm}^{-1}\right.$ instead of $5000-6000$ $\mathrm{cm}^{-1}$ ). Along with the observation that relative to the monomer emission the excimer emission is weak, this suggests that the pyrenyl moieties can not attain an optimal face-to-face geometry. The reason for this can be a large pyrenyl-pyrenyl distance and/ or limited ring overlap. In a geometry with a boat ring structure and axial substituents, the spatial separation between the pyrenyl groups is approximately $4.0 \AA$. This distance is not much larger than the optimal excimer interchromophore distance of 3.4-3.5 $\AA$. It is therefore likely that the weak excimer emission of relatively high energy is the result of reduced overlap of the pyrenyl $\pi$-systems in 2 .

In order to obtain a better understanding of the dynamics and energetics of excimer formation, a global target analysis $[8,14]$ of the streak camera fluorescence data of $\mathbf{2}$ was performed. The data were fitted with a number of two-state equilibrium models (Fig. 5), the various models differing in the initial assumptions. In all fits, the excimer state was assumed not to emit at wavelengths shorter than $390 \mathrm{~nm}$, which is justified by the difference steady-state fluorescence spectrum of $\mathbf{2}$ and $\mathbf{1}$ described above. For all models treated in the following, the quality of the fits was very good.

At first sight, an attractive option is to take $k_{11}$ equal to the decay rate of $\mathbf{1}$. However, this approach yielded a markedly positive free energy of excimer formation of $\Delta G_{\mathrm{EF}}=+25 \mathrm{meV}$, which rules out that it is appropriate. In contrast, sound results were obtained by assuming $\Delta G_{\mathrm{EF}}=0$, i.e. the driving force for excimer formation is zero. This approach gave $k_{11}=0.0190 \mathrm{~ns}^{-1}, k_{12}=k_{21}=$ $0.0254 \mathrm{~ns}^{-1}, \quad k_{22}=0.0208 \mathrm{~ns}^{-1}$ and a quantum

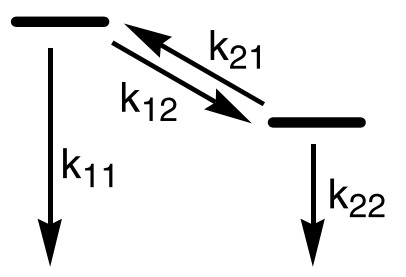

Fig. 5. Two-state equilibrium model used in the global target analysis of the time-resolved fluorescence of $\mathbf{2}$ in cyclohexane. The upper and lower state represent the locally excited pyrene and excimer state, respectively. yield for excimer formation of $\Phi_{\mathrm{EF}}=0.57$. Species Associated Spectra (SAS) obtained with these rate constants are fully plausible (Fig. 4b). The excimer emission is characterized by a symmetric gaussian with a maximum at $465 \mathrm{~nm}$ and a width of $3720 \mathrm{~cm}^{-1}$. The most negative $\Delta G_{\mathrm{EF}},-29 \mathrm{meV}$, was obtained in a model in which $k_{11}$ was set to 0 . This approach, which also yielded satisfactory SAS, can be regarded as a limiting case. It implies that the driving force of excimer formation is small in any case, and we consider the $\Delta G_{\mathrm{EF}}=0$ model to be representative. Other $\Delta G_{\mathrm{EF}}$ values are for instance $-177 \mathrm{meV}$ for the intermolecular pyrene excimer [15] and some $-100 \mathrm{meV}$ for 1,3-dipyrenylpropanes [16]. The small driving force for $\mathbf{2}$ is in accordance with its weak steady state excimer emission of relatively high energy.

It is also of interest to compare the rate of folding of $\mathbf{2}$ with that of other intramolecular excimers. Unfortunately, detailed kinetic models as presented above are not always available. However, as formation of the excimer in the examples below is essentially quantitative the reciprocal of the fluorescence risetime provides a proper estimate of the excimer formation rate. Suitable reference data are rates of $0.27 \mathrm{~ns}^{-1}$ for $1,3-\mathrm{di}(1-$ pyrenyl)propane in hexane [17], and about $0.5 \mathrm{~ns}^{-1}$ for dipyrenyl substituted linear tri- and tetrasilane in isooctane [5]. Thus, excimer formation in $\mathbf{2}$ is an order of magnitude slower than in other intramolecular excimers. This is not surprising since a cyclic system has less possibilities for conformational changes than a linear chain, i.e. the pre-exponential factor in the Arrhenius equation is smaller. The activation energy is not important in accounting for the slower kinetics, since energy barriers for geometry changes of cyclohexasilanes are similar to those of $n$-alkanes $[18,19]$. Nevertheless, this flexibility of the silicon ring must play an important role in the formation of the excimer. The result that $k_{12}=k_{21}$ can be interpreted by assuming that the silicon ring is undergoing conformational changes at this rate, thereby moving the pyrenyl substituents in and out of a position in which they give excimer emission. In this respect, the situation is different from the folding process of cyclic systems in the charge separated excited state of electron donor-acceptor molecules [8,14,20,21], which 
is driven by the coulombic attraction of oppositely charged chromophores. As conformational barriers in cyclohexasilanes are much lower than those in cyclohexanes it would be interesting to investigate whether dipyrenyl substituted cyclohexanes are also able to undergo intramolecular excimer formation.

Although significant changes in the fluorescence spectrum of $\mathbf{1}$ and $\mathbf{2}$ did not occur when changing from cyclohexane to solvents of medium polarity, in acetonitrile a different behaviour is observed (Fig. 2). For both 1 and 2 a broad structureless fluorescence is seen, with maxima at 438 (1) and 431 (2) $\mathrm{nm}$. In both cases, the local emission is almost completely quenched and the fluorescence intensity is much smaller than in nonpolar solvents. Fluorescence lifetimes are short $\left(\tau_{\mathrm{fl}} \leqslant 2 \mathrm{~ns}\right)$, and no time-dependent shifts occur (results not shown). A concentration effect was not observed. Since the broad emission is also present for $\mathbf{1}$ intramolecular excimer formation can not be involved. It then appears that the broad emission in acetonitrile originates from a $\sigma(\mathrm{Si}-\mathrm{Si}) \rightarrow 2 \mathrm{p} \pi^{*}$ charge separated (CS) state, which is formed by photoinduced electron transfer from the cyclohexasilane ring to the pyrenyl moiety. Similar intramolecular exciplex phenomena have been reported for phenylpentamethyldisilanes and pyrenyl substituted linear hexasilanes [3-5].

The formation of a $\sigma(\mathrm{Si}-\mathrm{Si}) \rightarrow 2 \mathrm{p} \pi^{*} \mathrm{CS}$ state in acetonitrile is supported by the redox potentials of 1 as determined by cyclic voltammetry. An irreversible reduction wave at $-2.13 \mathrm{~V}$ vs. SCE is observed for the pyrenyl acceptor, while the $\mathrm{Si}_{6} \mathrm{Me}_{11}$ moiety exhibits irreversible oxidation waves at +0.95 and $+1.19 \mathrm{eV}$ vs. SCE. These oxidation potentials are significantly lowered with respect to the oxidation potentials of dodecamethylcyclohexasilane, for which values of +1.31 and $+1.65 \mathrm{~V}$ vs. SCE were measured. This is again indicative of substantial $\sigma-\pi$ mixing. The redox potentials can be used to obtain the free energy of charge separation $\Delta G_{\mathrm{CS}}$ via [22]

$$
\begin{aligned}
\Delta G_{\mathrm{CS}}= & e\left(E_{1 / 2}^{\mathrm{ox}}-E_{1 / 2}^{\mathrm{red}}\right)-E_{00}-\frac{e^{2}}{4 \pi \varepsilon_{0} \varepsilon_{\mathrm{s}} R_{\mathrm{DA}}} \\
& -\frac{e^{2}}{8 \pi \varepsilon_{0}}\left(\frac{1}{r_{\mathrm{d}+}}+\frac{1}{r_{\mathrm{a}-}}\right)\left(\frac{1}{35.9}-\frac{1}{\varepsilon_{\mathrm{s}}}\right) .
\end{aligned}
$$

A zero-zero excitation energy of $E_{00}=3.21 \mathrm{eV}$ and a donor-acceptor distance of $R_{\mathrm{DA}}=6.6 \AA$, taken from the crystal structure of 2 [6], were used. Effective radical cat- and anion radii $r_{\mathrm{d}+}(5.3 \AA)$ and $r_{\mathrm{a}-}(4.0 \AA)$ were obtained by $r=\left(3 V_{\mathrm{m}} / 4 \pi\right)^{1 / 3}$, with the molecular volumes $V_{\mathrm{m}}$ adapted from the crystal structures of dodecamethylcyclohexasilane [23] and pyrene [24]. Application of these data reveals that in acetonitrile (dielectric constant $\left.\varepsilon_{\mathrm{s}}=35.9\right)$ charge separation is allowed since $\Delta G_{\mathrm{CS}}=-0.16 \mathrm{eV}$. In contrast, in cyclohexane $\left(\varepsilon_{\mathrm{s}}=2.02\right)$ it is forbidden by $0.24 \mathrm{eV}$, which is in agreement with the fluorescence data. Thus, in addition to surprising intramolecular excimer emission, intramolecular exciplex emission can also be observed for 2 .

\section{References}

[1] R.D. Miller, J. Michl, Chem. Rev. 89 (1989) 1359.

[2] R. West, in: E.W. Abel, F.G.A. Stone, G. Wilkinson (Eds.), Comprehensive Organometallic Chemistry II, vol. 2, Pergamon Press, Oxford, 1995, p. 77.

[3] H. Sakurai, H. Sugiyama, M. Kira, J. Phys. Chem. 94 (1990) 1837.

[4] D. Declercq, F.C. De Schryver, R.D. Miller, Chem. Phys. Lett. 186 (1991) 467.

[5] D. Declercq, P. Delbeke, F.C. De Schryver, L. Van Meervelt, R.D. Miller, J. Am. Chem. Soc. 115 (1993) 5702.

[6] V.E.M. Kaats-Richters, T.J. Cleij, L.W. Jenneskens, M. Lutz, A.L. Spek, C.A. van Walree, to be submitted.

[7] C.A. van Walree, M.R. Roest, W. Schuddeboom, L.W. Jenneskens, J.W. Verhoeven, J.M. Warman, H. Kooijman, A.L. Spek, J. Am. Chem. Soc. 118 (1996) 8395.

[8] X.Y. Lauteslager, I.H.M. van Stokkum, H.J. van Ramesdonk, D. Bebelaar, J. Fraanje, K. Goubitz, H. Schenk, A.M. Brouwer, J.W. Verhoeven, Eur. J. Org. Chem. (2001) 3105 .

[9] J.M. Haider, M. Chavarot, S. Weidner, I. Sadler, R.M. Williams, L. De Cola, Z. Pikramenou, Inorg. Chem. 40 (2001) 3912

[10] F.C. de Schryver, N. Boens, J. Put, Adv. Photochem. 10 (1977) 359.

[11] N.J. Turro, Modern Molecular Photochemistry, Benjamin/ Cummings, Menlo Park, 1978.

[12] C. Westermeier, H.-C. Gallmeier, M. Komma, J. Daub, Chem. Comm. (1999) 2427.

[13] K. Zachariasse, W. Kühnle, Z. Phys. Chem. Neue Folge 101 (1976) 267.

[14] I.H.M. van Stokkum, T. Scherer, A.M. Brouwer, J.W. Verhoeven, J. Phys. Chem. 98 (1994) 852. 
[15] A. Gilbert, J. Baggot, Essentials of Molecular Photochemistry, Blackwell, Oxford, 1991.

[16] K.A. Zachariasse, G. Duveneck, J. Am. Chem. Soc. 109 (1987) 3790.

[17] O.E. Zimmerman, R.G. Weiss, J. Phys. Chem. A 102 (1998) 5364.

[18] M.K. Leong, V.S. Mastryukov, J.E. Boggs, J. Phys. Chem. 98 (1994) 6961.

[19] N. Casarini, L. Lunazzi, A. Mazzanti, J. Org. Chem. 63 (1998) 9125.
[20] J.W. Verhoeven, T. Scherer, R.J. Willemse, Pure Appl. Chem. 65 (1993) 1717.

[21] F.J. Hoogesteger, C.A. van Walree, L.W. Jenneskens, M.R. Roest, J.W. Verhoeven, W. Schuddeboom, J.J. Piet, J.M. Warman, Chem. Eur. J. 6 (2000) 2948.

[22] A. Weller, Z. Phys. Chem. 133 (1982) 93.

[23] H.L. Carrell, J. Donohue, Acta Cryst. B 28 (1972) 1566.

[24] R. Allmann, Z. Kristallogr, Kristallgeom. Kristallphys. Kristallchem. 132 (1970) 129. 